\title{
The Incidence of Penicillin-sensitive Variant Colonies in Penicillinase-producing Strains of Staphylococcus pyogenes
}

\author{
BY MARY BARBER \\ From the Postgraduate Medical School of London and St Thomas's \\ Hospital Medical School
}

SUMMARY: Penicillinase-producing strains of Staphylococcus pyogenes isolated from human infections and kept under various conditions were plated out and fifty colonies from each plate tested for penicillin-sensitivity to determine the permanence of their resistance to penicillin.

Of 32 strains kept in Lemco broth and tested once, 5-12 months after isolation, two yielded only penicillin-sensitive colonies and fifteen others yielded a proportion of such.

Of six strains preserved by the gelatin-ascorbic acid drying process and kept for over a year, only one yielded penicillin-sensitive colonies.

Of six strains kept in Lemco broth with and without regular subculture and tested at intervals for 9 months, all gave rise to at least one penicillin-sensitive variant colony and more than half the colonies of one strain became penicillin-sensitive.

The natural tendency of these strains to yield penicillin-sensitive variant colonies was not appreciably accelerated by treatment with $\mathrm{X}$-rays or by growing them with other organisms.

From many studies on penicillin-resistant staphylococci it is clear that the cocci display two fundamentally different types of resistance; this was first pointed out by Spink, Hall \& Ferris (1945) and has been confirmed by many workers since. First, there is the type of resistance acquired in vitro by serial subculture of staphylococci in increasing concentrations of penicillin. The organisms often show gross changes in morphology, cultural characteristics and virulence, and tend to revert to the typical penicillin-sensitive variety after repeated subculture in the absence of penicillin. The resistance is not usually associated with the production of penicillinase. Bellamy \& Klimek (1948), however, describe a strain trained in vitro to grow in penicillin ' $4 \mathrm{mg} . / \mathrm{ml}$.', which produced small amounts of penicillinase, but only when grown in the presence of penicillin. This strain had lost many of the properties of a staphylococcus. It consisted of Gram-negative cocci and rods and had lost the ability to ferment lactose, sucrose, maltose, mannitol and galactose. The second type of resistance occurs in strains isolated from infective processes and human carriers; these strains do not differ from typical penicillin-sensitive strains of Staphylococcus pyogenes in morphology, cultural characteristics or virulence. As first pointed out by Kirby (1944), this type of resistance is characterized by the production of a powerful penicillin-inactivator. In reports by Spink et al. (1945) Bondi \& Dietz (1945), Gots (1945), Barber (1947a, b) and Barber \& Rozwadowska-Dowzenko (1948), all penicillin-resistant strains of Staph. pyogenes isolated in vivo were shown to destroy penicillin.

During the past two years penicillin-resistant strains of Staph. pyogenes 
were isolated at Hammersmith Hospital from 111 patients with staphylococcal infection, and a number were obtained from nasal and skin carriers (Barber, $1947 a, b$; Barber \& Rozwadowska-Dowzenko, 1948). All these strains were able to destroy penicillin. Single colonies of penicillin-resistant cocci appearing on the original culture plates of human material were subcultured into Lemco broth and maintained in the laboratory, many of them for from 9 to 18 months. In the present investigation forty-four of these cultures were studied to determine the permanence or impermanence of their resistance to penicillin.

\section{METHODS}

Penicillin ditch-plates. Ditches were cut at one side of Lemco agar plates and filled with the same medium containing approximately 10 units penicillin $/ \mathrm{ml}$. The ditch-plates were prepared $24 \mathrm{hr}$. or more before use to allow some penicillin to diffuse out of the ditch. As a control, the Oxford staphylococcus was streaked across every ditch-plate at the time of its use.

Penicillinase production was tested for by the method of Barber (1947a).

Typing by bacteriophage. Parent and variant cultures were phage-typed by Dr Allison by the method of Wilson \& Atkinson (1945).

\section{RESULTS}

Variants from thirty-two strains kept in Lemco broth for 5-12 months. Thirtytwo strains kept, with occasional subculture, in Lemco broth were plated out on Lemco agar plates and from each plate fifty colonies were picked off and streaked across penicillin ditch-plates. The results are summarized in Table 1. Of the 32 strains tested seventeen gave penicillin-sensitive colonies and from

Table 1. Distribution of percentage of colonies of penicillin-sensitive Staphylococcus pyogenes in thirty-two penicillin-resistant strains (fifty colonies of each strain tested.)

$\left.\begin{array}{rc}\text { No. of strains } & \text { Percentage } \\ 2 \\ 5 \\ 3 \\ 3 \\ 1 \\ 3\end{array}\right\} \begin{array}{cc}100 \\ 17 & 22-50 \\ 15 & 10-20 \\ & 4 \\ & 2 \\ & 0\end{array}$

two cultures all fifty colonies tested were penicillin-sensitive. All the penicillinsensitive colonies appeared to be as sensitive as the Oxford staphylococcus and all the resistant colonies grew across the ditch (Pl. 1, fig. 1). No intermediate degrees of resistance were encountered. The penicillin-sensitive colonies bred true on subculture and proved to have lost their capacity to destroy penicillin.

The parent broth cultures were then plated directly on to penicillin ditchplates. The two cultures from which only penicillin-sensitive colonies were obtained (Pl. 1, fig. 2) showed no penicillin-resistant colonies even by this method, which can detect as few as one in 10,000 resistant colonies (see Barber, 
$1947 b$ ). The fifteen cultures yielding a mixture all resembled wholly penicillinresistant strains and no colonial variation was detectable (Pl. 1, fig. 3). This is presumably because a few resistant organisms produce penicillinase sufficient to permit growth of the penicillin-sensitive organisms. In artificial mixtures of penicillin-sensitive and penicillin-resistant organisms the ratio of sensitive to resistant had to be at least 100:1 if the sensitive organisms were to be detected on a penicillin ditch-plate (PI. 1, fig. 4). One interesting point is that whereas penicillin-sensitive colonies get smaller as the penicillin is approached, the penicillin-resistant colonies get larger. This is particularly well seen in Pl. 1, fig. 4.

Variants from six strains preserved by the gelatin-ascorbic acid drying process. Six strains kept for over a year after drying by Stamp's (1947) method yielded far fewer variants. From five of the cultures no penicillin-sensitive colonies were isolated and one strain yielded only three.

Table 2. Incidence of penicillin-sensitive variants in six strains kept under controlled conditions for 9 months

No. of penicillin-sensitive colonies out of fifty tested

\begin{tabular}{|c|c|c|c|c|c|c|c|c|c|c|c|c|}
\hline \multirow[b]{2}{*}{ Tested after } & \multicolumn{2}{|c|}{$\overbrace{}^{\text {D3R }}$} & \multicolumn{2}{|c|}{$\overbrace{}^{\mathrm{D} 6 \mathrm{R}}$} & \multicolumn{2}{|c|}{$\overbrace{}^{\text {P193R }}$} & \multicolumn{2}{|c|}{ P268R } & \multicolumn{2}{|c|}{ Sp $249 R$} & \multicolumn{2}{|c|}{ P5155RR } \\
\hline & $a$ & $b$ & $a$ & $b$ & $a$ & $b$ & $a$ & $b$ & $a$ & $b$ & $a^{*}$ & $b$ \\
\hline 6 weeks & 0 & 0 & 0 & 0 & 0 & 0 & 1 & 0 & 13 & 5 & 6 & 1 \\
\hline 3 months & 0 & $\mathbf{0}$ & 0 & 1 & 0 & 0 & 0 & 0 & 9 & 3 & 5 & 0 \\
\hline 4 months & $\mathbf{0}$ & 0 & 0 & 1 & $\mathbf{0}$ & o & 0 & 0 & 28 & 12 & 3 & 0 \\
\hline 5 months & 0 & 0 & 0 & 0 & 0 & 0 & 3 & 1 & 25 & 20 & $\mathbf{5}$ & $\mathbf{0}$ \\
\hline 6 months & 0 & 0 & 0 & 1 & 0 & 0 & 0 & 1 & 36 & 25 & 4 & 3 \\
\hline 9 months & 0 & 1 & 0 & - & 1 & 0 & 0 & 0 & 34 & 40 & 41 & 6 \\
\hline 12 months & 0 & 0 & 0 & - & $\mathbf{0}$ & 0 & $\mathbf{0}$ & - & 42 & 31 & $\mathbf{5 0}$ & 11 \\
\hline
\end{tabular}

$a=$ Subcultured twice a week in Lemco broth.

$b=$ Left on bench in Lemco broth and subcultured every 3 months.

- = Not tested.

* This culture became contaminated with a bacillus between the fourth and fifth months.

Variants from six strains kept under controlled conditions for 9 months. Six cultures were studied more carefully over a period of 9 months; five of these had been isolated within a few weeks of starting the experiment and one, P5155RR, was a penicillin-resistant colony from a culture isolated 7 months previously and at that time yielding a mixture of sensitive and resistant colonies. One culture of each strain was subcultured twice a week in Lemco broth, and one was left on the bench in the same medium and subcultured once during the period. Each of the two series was plated out after $1 \frac{1}{2}, 3,4,5,6$, 9 and 12 months; and, except for two strains left without subculture that had died at 9 and 12 months, fifty colonies from each strain were tested on the ditch-plates. In all 600-700 colonies were tested from each of the twelve cultures (Table 2). From all six strains penicillin-sensitive variant colonies were isolated, although two yielded only a single variant and two more only a few. Strain Sp249R yielded an increasing number of penicillin-sensitive 
colonies and subculture seemed to increase the rate of conversion. For the first 6 months strain P5155 RR, when subcultured, gave a few variant colonies at each test. At the ninth month, however, there were forty-one colonies and at the twelfth fifty. This specimen became contaminated with a bacillus between the fourth and fifth months. When left on the bench the strain yielded only one variant in the first 5 months and then at subsequent tests a steadily increasing proportion.

As in the previous experiment, nearly all the colonies tested were either as sensitive as the Oxford staphylococcus or grossly resistant, growing right across the penicillin ditch. Occasionally, however, colonies showed an intermediate degree of resistance; but when such streaks were emulsified in broth, plated on plain agar and fifty colonies again tested, a mixture of penicillinsensitive and resistant organisms was found. As before, penicillin-sensitive colonies bred true and had lost the capacity to destroy penicillin.

\section{Attempts to stimulate variation}

Two agents were tested for power to accelerate the natural tendency of penicillinase-producing staphylococci to give rise to penicillin-sensitive variants.

The effect of $X$-radiation. A dose of $1,000,000 \mathrm{r}$. (about the maximum dose leaving any surviving staphylococci) was used on strains D3 and D6, which naturally produced only occasional penicillin-sensitive variants. The irradiated cultures were plated out after 1, 2, 6, 12, 20 and 26 subcultures and fifty colonies examined. Of the 300 colonies tested from each after $\mathrm{X}$-raying, none was penicillin-sensitive.

The effect of mixing cultures with Streptococcus pyogenes. Following the work of Voureka (1948), Streptococcus pyogenes (Group A) was mixed with penicillinresistant staphylococci. Three strains of Strep. pyogenes, including the strain Milne used by Voureka, and ten strains of staphylococci, some of which were producing many penicillin-sensitive variants and some only producing a few, were used. The streptococci were grown for 18-24 hr. in Hartley broth and the cultures then seeded with two drops of a broth culture of one of the staphylococci. The mixtures were incubated for $24 \mathrm{hr}$., plated and fifty staphylococcal colonies tested. Control cultures of Hartley broth, seeded with a staphylococcus only, were similarly treated. There was (Table 3) no obvious difference in the number of variants occurring in the mixed cultures and in the controls.

The effect of mixing penicillin-resistant and penicillin-sensitive strains of Staph. pyogenes. A penicillin-sensitive (S) strain and a penicillin-resistant (R) strain (D3) were mixed in the following proportions: S/R 1:1, 1:100, $100: 1$. The mixtures were divided into two parts, one subcultured twice a week in Lemco broth and the other left on the bench in the same medium. At varying intervals during 5 months the mixtures were tested (Table 4 ). The S/R 1:1 mixture subcultured twice a week yielded penicillin-sensitive colonies in proportions varying from $1: 1$ to $4: 1$, whereas the culture left on the bench gave an increasing number of penicillin-sensitive colonies until all fifty tested were sensitive. Even at this stage, however, when the culture was plated directly on 
to a penicillin ditch-plate, a few resistant colonies were isolated. The $S / R$ 1:100 mixture when subcultured became and remained a nearly equal mixture and when left on the bench yielded a few sensitive colonies for the first 4 months and suddenly at the fifth month became predominantly penicillin-sensitive. With

\section{Table 3. Incidence of penicillin-sensitive variants after mixing with Streptococcus pyogenes}

\begin{tabular}{|c|c|c|c|c|c|}
\hline \multirow[b]{3}{*}{ Strain } & \multicolumn{5}{|c|}{ No. of penicillin-sensitive colonies out of fifty tested } \\
\hline & \multicolumn{3}{|c|}{ Mixed with Strep. pyogenes, strain } & \multicolumn{2}{|c|}{ Control cultures } \\
\hline & Richards & C203 & Milne & 1 & 2 \\
\hline D3 $\mathbf{R}$ & 1 & 0 & o & o & 0 \\
\hline D $6 \mathrm{R}$ & 0 & . 0 & 0 & 0 & 0 \\
\hline Sp 249 R & 43 & 41 & 32 & 36 & 44 \\
\hline P5155 RR & 15 & 10 & 15 & 10 & 9 \\
\hline P6395R & 6 & 4 & - & 8 & - \\
\hline Broor R & 14 & 16 & - & 18 & - \\
\hline Pro04 R & 29 & 26 & - & 21 & - \\
\hline P7108 R & 12 & 20 & - & 33 & - \\
\hline P268R & - & $\ldots$ & 0 & 0 & - \\
\hline P193R & - & - & 0 & 1 & - \\
\hline
\end{tabular}

Table 4. Incidence of penicillin-sensitive colonies in mixtures of penicillinsensitive and penicillin-resistant strains of Staphylococcus pyogenes

No. of penicillin-sensitive colonies out of fifty tested

\begin{tabular}{|c|c|c|c|c|c|c|c|}
\hline \multirow[b]{2}{*}{ Tested after } & \multicolumn{2}{|c|}{ S/R $1: 1$} & \multicolumn{2}{|c|}{$S / R 1: 100$} & \multicolumn{2}{|c|}{$\mathrm{S} / \mathrm{R} 100: 1$} & \multirow{2}{*}{$\begin{array}{c}\text { S/R } 1: 3 \\
\text { X-irradiation }\end{array}$} \\
\hline & $a$ & $b$ & $a$ & $b$ & $a$ & $b$ & \\
\hline (Immediately) & 19 & 34 & 4 & - & 49 & - & 49 \\
\hline 2 weeks & 一 & - & $\mathbf{2}$ & 1 & 50 & 50 & 39 \\
\hline 4 or 6 weeks & 29 & 47 & 23 & 3 & 44 & 50 & 16 \\
\hline 2 months & 17 & 48 & 34 & 6 & 41 & 50 & 20 \\
\hline 3 months & 10 & 49 & 26 & $\mathbf{3}$ & $\begin{array}{l}49 \\
48\end{array}$ & $\begin{array}{l}39 \\
39\end{array}$ & 34 \\
\hline 4 months & 14 & 50 & 20 & 7 & $\begin{array}{l}35 \\
34\end{array}$ & $\begin{array}{l}14 \\
11\end{array}$ & 48 \\
\hline 5 months & 16 & $50 *$ & 21 & 42 & $50 *$ & 30 & 50 \\
\hline
\end{tabular}

the S/R 100:1 mixture once again there appeared to be an increase in the strain originally in the minority. The results obtained at the third and fourth months were surprising and were therefore repeated, but with similar results. The results with these mixtures are difficult to interpret. One explanation would be that the two strains grow at different rates. It is, however, possible that a transference of properties with regard to penicillin-sensitivity takes place.

The S/R 1:1 mixture was also subjected to $\mathrm{X}$-irradiation of 1,000,000 $\mathrm{r}$. to see whether this would affect one strain more than the other. The $\mathrm{X}$-irradiated 
mixture was then subcultured twice a week in Lemco broth for 6 months. It will be seen from Table 4 that the number of resistant colonies was diminished immediately, that it increased until the second month, after which it steadily decreased until at 6 months no resistant colonies were detected even when the culture was plated directly on to a penicillin ditch-plate. It seems probable that the sensitive strain was originally more susceptible to the lethal action of irradiation, but that serial subculture, as with the S/R 100:1 mixture not irradiated, led to the sensitive colonies, originally in the minority, eventually becoming predominant.

\section{Typing by bacteriophage}

Since the cultures used in these experiments were derived originally from single colonies, not single cells, the purity of eight strains was checked by comparing the phage reaction of parent cultures, the variants, and cultures obtained from penicillin-resistant descendant colonies. In all eight strains, the variants were of the same phage type as the parent, and as the penicillinresistant daughter colonies.

\section{Colonial variations}

A number of variations in colonial appearance was observed during the course of these experiments, especially in cultures which had been in the laboratory for some time. The variant colonies were best seen when plates were incubated for 18-24 hr. and then left on the bench for a few days. Gross variations in colour and opacity occurred and were apparently favoured by repeated subculture, so that a single plate from a subcultured strain often consisted of typical golden, white, semi-transparent and transparent colonies. Size variations were also very common and were more marked in plates from the cultures that had been left on the bench without subculture, many consisting of a large number of small pin-point colonies with a few larger and more typical ones (Pl. 1, fig. 5). Another, less frequent variant occurring in several strains was a colony with an irregular and crenated edge, a rough or matt surface and a depressed centre (Pl. 1, fig. 6). All these colonial variants retained their capacity to clot citrated or oxalated plasma. Occasionally coagulase-negative colonies were encountered, but these were discarded as probable air contaminants. There was no association between colonial appearance and sensitivity to penicillin.

\section{DISCUSSION}

Studies on the permanence of resistance to penicillin of penicillinase-producing staphylococci have in many cases been invalidated by the assumption that the individual organisms making up a culture act uniformly in their behaviour to penicillin. Thus Blair, Carr \& Buchman (1946) and Spink \& Ferris (1947), as a result of testing the whole culture, claimed that cultures of penicillinaseproducing staphylococci were permanently resistant to penicillin. But, since a few penicillinase-producing cocci may protect many penicillin-sensitive cocci in the culture from the action of penicillin (see Pl. 1, fig. 3), testing the whole culture does not give an accurate picture of what is really happening. 
The present study makes it clear that penicillinase-producing strains of Staph. pyogenes tend to give rise to penicillin-sensitive variants when kept in the laboratory. It is, however, obvious that the method used is not accurate enough for much reliance to be placed on the actual number of variants obtained at any one time. Because single colonies, not single cells, were the starting-point for all experiments, it is possible that the original cultures consisted of a mixed population. The identity of phage-type of parent and variant colonies of eight strains, however, make it almost certain that at least in these strains, the penicillin-sensitive colonies were true variants of a penicillin-resistant parent.

I have not yet found a method of accelerating the natural variation, but this line of investigation is being continued. Voureka (1948) claims to have rendered both types of penicillin-resistant staphylococci sensitive to penicillin by mixing them with other organisms, such as the Milne streptococcus. My results (Table 3) do not confirm this. Bennison \& Schwabacher (1948) have also been unable to repeat Voureka's results. What happens when two strains of Staph. pyogenes are mixed is not clear. In both $S / R 1: 100$ and S/R 100:1 mixtures there was an increase in the minority strain. It is, however, possible that the strain in the majority was giving rise to variant colonies.

Until we discover the mode of origin of penicillin-destroying strains of staphylococci, it is not possible to understand the mechanism whereby they give rise to penicillin-sensitive variant colonies. Three explanations have been put forward. The first is that this type of resistance is truly acquired by contact with penicillin. As pointed out by Luria (1947), this would represent an example of the inheritance of an acquired characteristic, and therefore is contrary to the 'general outlook of modern biological thought'. The second, suggested by Barber $(1947 a)$, is that a few strains of Staph. pyogenes have always been penicillin-resistant and are now increasing in frequency by a simple process of selection. This view is supported by the fact that so far it has not proved possible to produce this type of resistance to penicillin in vitro. The frequency, however, with which these strains yield penicillin-sensitive variants is against this hypothesis. The third view, first put forward by Demerec (1945) to explain the origin of ' acquired' penicillin-resistance in vitro by staphylococci, and later by Spink \& Ferris (1947) as an explanation for the origin of penicillinaseproducing strains, is that these resistant organisms arise originally as rare mutants independent of the presence or absence of penicillin. In the presence of the antibiotic, however, these mutants are favoured at the expense of the penicillin-sensitive parent and thus increased in frequency by differential selection. If this hypothesis be correct then the penicillin-sensitive variants recorded here must be regarded as reverse mutants. Their frequency, in view of the difficulty of demonstrating the direct mutation, is perhaps surprising, but as Luria (1947) says 'very mutable characters often present rates of reverse mutations higher than the direct mutation rates'.

My thanks are due to Dr V. D. Allison for the phage-typing results, to Mr E. V. Willmott for the photographs, and to Dr A. Voureka for supplying me with a culture of the Milne streptococcus. 


\section{REFERENCES}

Barber, Mary (1947a). Coagulase-positive staphylococci resistant to penicillin. J. Path. Bact. 59, 373.

Barber, Mary (1947b). Staphylococcal infection due to penicillin-resistant strains. Brit. med. J. ii, 863.

Barner, Mary \& Rozwadowska-Dowzenko, Marie (1948). Infection by penicillinresistant staphylococci. Lancet, ii, 641.

Bellamy, W. D. \& KıмmeK, J. W. (1948). Some properties of penicillin-resistant staphylococci. J. Bact. 55, 153.

Bennison, W. H. \& Schwabacher, H. (1948). Sensitization of penicillin-resistant bacteria. Lancet, i, 885.

Blair, J. E., Carr, Miriam \& Buciman, J. (1946). The action of penicillin on staphylococci. J. Immunol. 52, 281.

Bondi, A. \& Dietz, Catherine (1945). Penicillin-resistant staphylococci. Proc. Soc. exp. Biol., N.Y., 60, 55.

Demerec, M. (1945). Production of staphylococcus strains resistant to various concentrations of penicillin. Proc. Nat. Acad. Sci. 31, 215.

Gots, J. S. (1945). Production of extracellular penicillin-inactivating substances associated with penicillin resistance in Staph. aureus. Proc. Soc. exp. Biol., N.Y., 60, 165.

Kirby, W. M. M. (1944). Extraction of a highly potent penicillin-inactivator from penicillin-resistant staphylococci. Science, 99, 452.

Luris, S. E. (1947). Recent advances in bacterial genetics. Bact. Rev. 11, 1.

Spink, W. W., Hall, W. H. \& Ferris, V. (1945). Clinical significance of staphylococci with natural or acquired resistance to the sulfonamides and to penicillin. J. Amer. med. Ass. 128, 555.

Spink, W. W. \& Ferris, V. (1947). Penicillin-resistant staphylococci. Mechanisms involved in the development of resistance. J. Clin. Invest. 26, $\mathbf{3 7 9}$.

Stamp, LoRD (1947). The preservation of bacteria by drying. J.gen. Microbiol. 1, 251.

Voureka, A. (1948). Sensitization of penicillin-resistant bacteria. Lancet, , 62.

Wilson, G. S. \& Atkinson, J. D. (1945). Typing of staphylococei by the bacteriophage method. Lancet, $\mathrm{i}, 647$.

\section{EXPLANATION OF PLATE}

Penicillin ditch-plates $\left(\times \frac{3}{4}\right)$

Fig. 1. Typical example of strain showing a mixture of penicillin-resistant and sensitive colonies. Central streak $=$ Oxford staphylococcus.

Fig. 2. Upper half plated with culture P6652, penicillin-resistant on primary isolation now sensitive, and lower half with the Oxford staphylococcus.

Fig. 3. Strain giving nine out of fifty penicillin-sensitive colonies-mixture undetectable by this method. Central streak $=$ Oxford staphylococcus.

Fig. 4. Mixture of penicillin-sensitive and resistant staphylococei in proportion of 100 sensitive to 1 resistant. Note gradual fading out of sensitive colonies as penicillin is approached and large resistant colonies near penicillin. Central streak $=$ Oxford staphylococcus.

\section{Colonial variations}

Fig. 5. One typical-sized colony and a number of small colony variants from same culture, $\times 20$.

Fig. 6. Two typical smooth colonies of Staph. pyogenes and three 'rough' variants, $\times 18$.

(Received 29 September 1947) 

Journal of General Microbiology, Vol. 3, No. 2

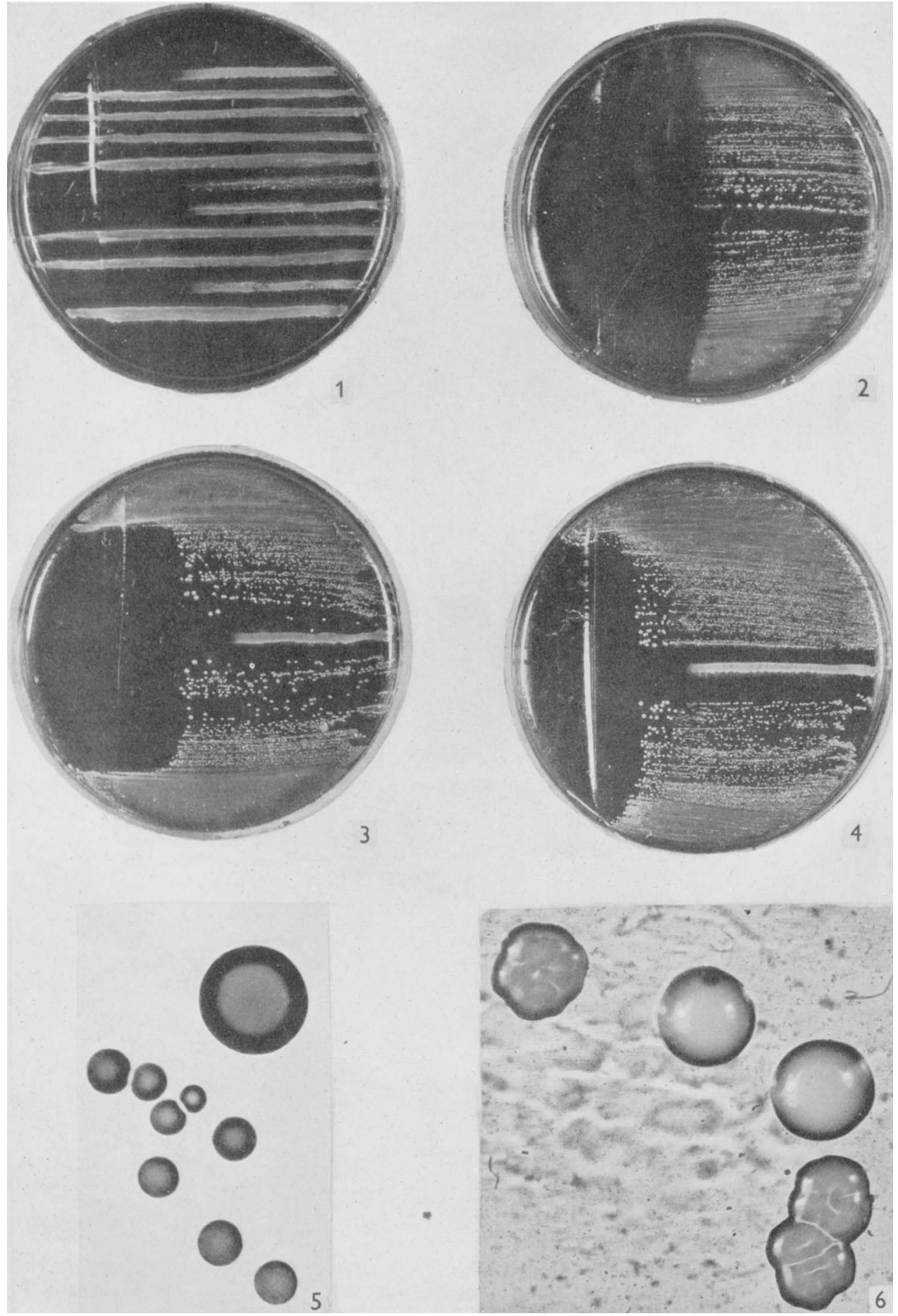

Figs. 1-6

Mary Barber - Variants of pleniclleinase-producing stapifylococci. Plate 1 